\section{EDP 13}

DILEMMA DER TUMORLOKALISATION NACH SEROLOGISCHER FRÜHDIAGNOSE: AUSWEG IN SICHT?

S. Matzku

Die Idee, der serologischen Diagnose erhöhter Tumormarker-Werte eine Immunszintigraphische Lokalisation unter Verwendung von Antikörpern gegen eben dieses Markermolekül folgen zu lassen, wurde am Fall des carcino-embyonalen Antigens (CEA) mit einigem Erfolg realisiert. Ahnliche Erfahrungen wurden in geringerem Umfang auch mit anderen Tumormarkern gesammelt. Allerdings ergibt sich in jedem Fall das Problem, daß nennenswerte Markerkonzentrationen im Blut den Antikörper blockieren und damit die szintigrafische Darstellung beeinträchtigen können. Diese Konstellation ist von prinzipieller Bedeutung, denn sie ist regelmäßig dann gegeben, wenn der Tumormarker sezerniert wird und mithin dem serologischen Nachweis zugänglich ist. Strategien zur Behebung der inhärenten Limitierung werden vorgestellt.

Für die Immunszintigrafie ist die gegentellige Ausgangslage, nämlich die stabile Verankerung des Markermoleküls an der Tumorzelle (ohne Serumspiegel) wesentlich günstiger. Dennoch kann die Methode nicht uneingeschränkt als Frühdiagnostikum angesehen werden, unter anderem weil die individuellen Schwankungen der Markerexpression einen $z u$ großen unsicherheitsfaktor darstellen und weil die Anrelcherung in kleinen Tumorknoten, bedingt durch die geringe Zugänglichkeit von solidem Tumorgewebe für große (Antikörper)Moleküle. für ein suffizientes Targeting oft nicht ausreicht.

Institut für Nuklearmedizin, Deutsches Krebsforschungszentrum, Pf. 101949, D-6900 Heidelberg

\section{EDP 14}

THE ROLE OF TUMOR MARKERS FOR SECOND-LOOK CANCER SURGERY U. Metzger, F. Largiadèr

Carcinoembryonic antigen (CEA) for colorectal cancer and Alphafetoprotein (AFP) for germ cell tumors, are the most commonly used tumor markers in surgery.

207 patients curatively resected for colorectal cancer have been followed in a prospective, controlled study using serial CEA, ultrasound, colonoscopy, chest-x-ray and clinical examination for early detection of recurrent tumor. After a median follow-up of 2,5 years, 52 recurrencies (258) have been detected, raising CEA value beeing the most sensitive indicator (86\%) among the diagnostic procedures. 48 patients (92\%) had no symptoms of their recurrence at the time of diagnosis. Median survival of 13 (25\%) patients reresected for cure has not yet been reached at 2,5 years, whereas median survival for patients not operated upon $(n=26)$ or incompletely resected $(n=13)$ is 8,5 months.

AFP was investigated in 30 patients with stage II $C$ and III non-seminomatous testicular cancer "who underwent surgery for residual tumor after intensive chemotherapy. AFP levels over $10^{4} \mathrm{ng} / \mathrm{ml}$ at diagnosis and persistently eleveted AFP values preoperatively were associated with failure of surgery to achieve complete remission ( $p<0.05$ ) and to achieve long-term survival even after surgical complete remission.

Both markers prooved to be sensitive indicators for recurrent or active tumor and were important guides for second-look operations.

Department of Surgery, University Hospital CH-8091 Zurich

\section{EDP 15}

ZYTOLOGISCHE FRÜHDIAGNOSTIK-WERTIGKEIT UND PROBLEMATIK DES VERFAHRENS H.Naujoks

Zellabstriche von Portio und Zervikalkanal erlauben verläßlich die Erkennung nicht nur des präklinischen Kollumkarzinoms sondern auch seiner Vorstufen(CIN I-III), Sie ermöglichen auch Angaben zu metaplastischen, regenerativen, entzündlichen und hormonabhängigen Epithelveränderungen und geben Hinweise auf virusbedingte Infektionen(Herpes, HPV). Ein zytologisches Screening kann zur Senkung der Mortalität beim Kollumkarzinom, zur Vorverlegung therapiezugängiger Krebsstadien und zur rechtzeitigen Eliminierung von Krebsvorstufen führen.

Probleme entstehen durch das Fehlen notwendiger Voraussetzungen fur ein erfolgreiches Screening: ungenigende Beteiligung an der gesetzlichen Krebsfruherkennung und Nichterfassung risikoreicher Gruppen;Fehler bei der Durchfïhrung zytologischer Untersuchungen(Entnahmefehler, Laborfehler); unzureichende Qualitätssicherung. So wird die Beteiligungsrate von $30-40 \%$ der berechtigten Frauen angefürt.Die Rate falsch negativer Zellabstriche wird mit bis zu $20 \%$ angegeben.Klinische Kollumkarzinome haben zwischen 11 und $30 \%$ unauffällige Voruntersuchungsbefunde in zytologischen Abstrichen gezeigt. Die ergänzende kolposkopische Untersuchung ist im Rahmen der gesetzlichen Krebsfrüherkennung nicht vorgesehen. Eine Qualitätssicherung ist nur bedingt durch die Datenerfassung der Untersuchungsbögen möglich.Probleme entstehen durch Verlagerung des zytologischen Screening in zu kleine Einheiten.

Abt.f.K1in.Zytologie, Universitäts-Frauenklinik Theodor-Stern-Kai 7,D60ooFrankfurt a.M.-70,BRD

\section{EDP 16}

Tragen bildgebende Verfahren zur Friherkennung maligner Tumoren bei?

Aus der Anamese und klinischen Untersuchung ergibt sich die Indikationsstellung für bildgebende Untersuchungen. Die Möglichkeiten der Friherkennung maligner Tumoren ist durch das physikalische Auflösungsvermögen der Untersuchungstechniken. die anatomische Lage der Tumoren und den Zeitpunkt des Einsatzes der Untersuchung gegeben. Während die Möglichkeiten der konventionellen Röntgenunter suchungen bei der Friherkennung von Tumoren der Lunge. der Knochen und des Gastrointestinaltraktes bekannt sind, wird die Wertigkeit der neueren Techniken (Ultraschall.Computertomographie. Magnetresonanztomographie) bei Tumoren des Gehirns. der weiblichen Brustdruise. der Lunge und des Herzens. bei Malignomen der Leber. des Pankreas sowie des Retroperitonealraumes und des Beckens an Beispielen aufgezejet. Die Differentialdiagnose gegenuber gutartigen Prozessen ist speziell bej kleinen Tumoren schwierig und kann letztich nur histologisch erfolgen. Unentbehrlich sind die bildgebenden Verfahren jedoch in der Lokalisation und Stadieneinteilung der Malignome und in der Nachsorge der Patienten. 